\title{
La importancia de la historia para comprender el trabajo y sus transformaciones
}

Marina Kabat ${ }^{1}$

\section{Resumo:}

Com base em pesquisas empíricas anteriores, revisamos o debate sobre o processo de trabalho marxista com base nas contribuições mais recentes de estudiosos argentinos e brasileiros. Afirmamos a importância de levar em conta o papel da natureza nas transformações do trabalho e suas diferentes temporalidades. Argumentamos que o esquecimento da influência da natureza no caminho das mudanças do processo de trabalho leva a concepções simplificadas e unilineares da organização do trabalho. Essas visões tendem a caracterizar a base técnica da sociedade capitalista em um determinado momento, baseado apenas no processo de trabalho predominante em um único setor da economia capitalista.

Palavras-chave: Processo de trabalho; desqualificação; taylorismo; marxismo; grande indústria; regulacionismo; lutas dos trabalhadores.

\section{The importance of history to understand labor and its transformations}

\begin{abstract}
:
On the basis of previous empirical research, we review the Marxist labor process debate focusing in the latest contributions from Argentine and Brazilian scholars. We state the importance of taking into account the role of nature in work's transformations and its different temporalities. We argue that the oblivion of the influence of nature in the path of the labor process' changes leads to simplified and unilineal conceptions of labor organization. These views tend to characterized the hole technical base of the capitalist society in a certain moment only upon the labor process prevalent in just a single sector of capitalist economy.
\end{abstract}

Key words: labor process; deskilling; Taylorism; Marxism; large scale industry; regulationism; workers' struggles.

\section{Algunas cuestiones metodológicas iniciales}

Trabajo es toda actividad humana orientada a un fin. En esa actividad, es decir en el proceso de trabajo, el hombre interactúa con la naturaleza y, al hacerlo, modifica la naturaleza y así mismo como parte de

${ }^{1}$ Professora da Universidad de Buenos Aires (UBA) e pesquisadora do Centro de Estudios e Investigación en Ciencias Sociales (CEICS). E-mail: marinakabat@yahoo.com.ar. 
ella. Mediante el trabajo el hombre actúa sobre y modifica el medio ambiente, al mismo tiempo que transforma su propia naturaleza humana. Como tempranamente planteó Engels, el trabajo hizo al hombre (ENGELS, 1975). ${ }^{2}$ Una primera derivación de esto es el carácter esencial del trabajo a la naturaleza humana $\mathrm{y}$, por ende, la centralidad del problema de su alienación.

De esta definición de proceso de trabajo surge que el mismo no es una actividad unilateral del ser humano. La naturaleza, ofrece resistencia a su transformación. Esta resistencia es diferente en distintas actividades. En artículos anteriores hemos defendido los estudios de proceso de trabajo por rama de actividad como propuesta metodológica capaz de superar al mismo tiempo estudios a nivel micro de empresas individuales y concepciones demasiado generales (SARTELLI; KABAT, 2014). Pero, esta propuesta no solo se fundamenta en la necesidad de escoger el nivel de análisis más adecuado para articular la descripción cualitativa y cuantitativa. El estudio por ramas económicas también se funda en las diversas temporalidades de las transformaciones de los procesos de trabajo, producto de estos diversos grados de resistencia que la naturaleza opone a su transformación por el trabajo humano. Por ejemplo, no avanza del mismo modo la transformación de actividades que pueden realizarse en forma masiva (moler el trigo, por ejemplo), frente a otras que requieren el trabajo humano sea ejercido sobre cada unidad (fabricación de ropa o automóviles, por ejemplo). Estos ejemplos muestran que los procesos de trabajo no mudan solo al ritmo de la inventiva y la voluntad humana, sino que estas chocan con la multiforme materialidad de la naturaleza, en algunos casos más maleable que en otros a la acción humana. Esto explica que ciertas ramas presenten un importante rezago respecto a otras en cuanto a la transformación de los procesos de trabajo.

Del mismo modo, los procesos por los cuales la naturaleza se transforma partir del trabajo humano, siendo como son procesos materiales, tienen una temporalidad determinada. Esto constituye una fuerte barrera a la modificación de los procesos de trabajo en ciertas ramas. El hombre debe violentar con el concurso de la ciencia esos tiempos naturales. Por ejemplo, debe modificar la producción de vegetales alterando los ritmos de germinación. Lo mismo ocurre con el curtido de cueros, secado de maderas, fabricación de quesos y vinos. Los primeros materiales con los que el hombre trabaja son los disponibles en la propia naturaleza, baste recordar los múltiples usos que la clara de huevo mantuvo, aún en el siglo veinte, en los más diversos procesos industriales.

2 Esta correcta tesis de Engels fue recuperada con mayor información científica por Stephen Jay Gould (1996). 
El desarrollo de la ciencia, en particular el desarrollo de la química permite la creación de nuevos materiales con procesos de actuación más limitados.

A diferencia de concepciones sociológicas en boga en diferentes momentos, Marx brinda un abordaje histórico a la transformación de los procesos de trabajo. Este enfoque es útil para comprender cualquier actividad laboral, desde la prostitución (LÓPEZ RODRÍGUEZ, en prensa), la fabricación de automóviles (HARARI, 2019; RODRIGUES DE MORAES NETO, 2003), la producción rural (SARTELLI; KABAT, 2009), la gastronomía (SARTELLI, 2001), las tareas de reciclaje informal de cartón (VILLANOVA, 2017). Este abordaje permite distinguir tanto las diferencias materiales en la forma de ejecutar el trabajo como los cambios que estas engendran en la relación capital trabajo (subsunción formal y real).

Como los conceptos marxistas son poco conocidos, y muchas veces hasta confundidos con los conceptos regulacionistas, en los próximos acápites reseñamos sus rasgos principales, ejemplificando con algunos estudios realizados en el grupo de investigación que coordinamos junto con Eduardo Sartelli. Pasamos luego a discutir las tendencias fundamentales de los procesos de trabajo bajo el capitalismo. Planteamos que las concepciones que se plantean como superadoras carecen de un adecuado entendimiento de los planteos de esta teoría y confunden aseveraciones de Braverman con las del propio Marx. Finalmente, sostenemos que tanto Braverman como los regulacionistas carecen de una adecuada comprensión histórica de los procesos que estudian, perspectiva que, en las conclusiones convocamos a recuperar.

\section{De la cooperación simple a la manufactura}

La primera forma que asume el trabajo bajo el capitalismo es la cooperación simple. 3 En principio, ésta implica sólo un cambio cuantitativo en tanto conduce a un aumento de los obreros que trabajan juntos para el mismo patrón efectuando las mismas tareas o algunas de naturaleza semejante. Sin embargo, a pesar de que no se modifica la forma de realizar el trabajo, operan ciertas transformaciones. Por un lado, en la jornada de trabajo de un grupo relativamente grande de obreros se compensan naturalmente las diferencias que puede haber entre ellos y, en conjunto, se obtiene una jornada de trabajo social medio.

A la vez, se revolucionan las condiciones objetivas del proceso de trabajo, mediante el uso colectivo de los medios de producción, lo que deriva en una economía de recursos. Al mismo tiempo, algunas actividades resultan más productivas, es decir consumen menos trabajo social al realizarse mediante la colaboración de muchos obreros, aunque no

3 En esta sección seguimos la formulación de Marx (MARX, 1999, t. I, v. 2, caps. 11-13). 
aparezca aun la división de tareas. Esto ocurre, por ejemplo, con el traslado de objetos, puesto que estos recorren el mismo espacio en menos tiempo al formarse una cadena humana. Con la cooperación de muchos asalariados se torna necesaria la función directiva. El capital ejerce esta función de un modo despótico. Como los obreros sólo cooperan tras haber vendido su fuerza de trabajo, el producto de esa cooperación no les pertenece; la fuerza productiva del trabajo social se transforma en fuerza productiva del capital y aparece como si brotara de éste.

Con la manufactura surge una nueva forma de cooperación, ya de tipo complejo, basada en la división del trabajo sistemática y permanente. Anteriormente, en forma ocasional podía establecerse alguna forma de división de tareas, pero esta no llegaba a constituir una forma fija. En cambio, con la manufactura, las tareas se dividen y asignan en forma permanente a distintas personas. Las personas fijadas a sus puestos de trabajo parcelarios se especializan, perfeccionándose en la tarea que realizan.

El obrero manufacturero, realiza con virtuosismo y velocidad su tarea, a la cual adapta su cuerpo y su psiquis, a costa de la pérdida de otras capacidades y habilidades. Ciertos músculos se hiperdesarrollan mientras otros se atrofian. El conjunto del cuerpo del obrero se transforma en virtud de su trabajo parcelario. Al mismo tiempo, el obrero que realiza una sola tarea usa una sola herramienta: la especialización del obrero conduce la especialización de las herramientas. Estas, son modificadas para servir mejor a una única actividad. En el período manufacturero, las herramientas se simplifican, diferencian y adaptan a las distintas operaciones parciales. Todo esto favorece el proceso ulterior de mecanización que, sin embargo, implica la negación del sistema de trabajo basado en el obrero manual y su herramienta.

Podemos encontrar dos tipos de manufactura: orgánica, cuando consiste en la unión de productos independientes, como en el caso de la producción de relojes, y heterogénea, cuando el producto debe pasar por una serie de procesos consecutivos, como en la fabricación de calzado. En ambas formas de manufactura, se asigna un determinado número de obreros para cada tarea parcial; reaparece así, dentro de éstas, la cooperación simple. El número de los obreros destinados a cada operación guarda una relación proporcional, calculada en base a la experiencia. Del mismo modo, se establece la cantidad de productos que cada una de estas secciones debe suministrar en un determinado tiempo, con lo que se logra una regularidad, continuidad e intensidad superior del trabajo. Estas características, convierten en una ley técnica del proceso de producción el que el capitalista obtenga de sus obreros el trabajo socialmente necesario. 
La manufactura emplea, ocasionalmente, maquinaria, generalmente para trabajos masivos que requieren gran energía, pero ésta sigue siendo un elemento secundario dentro del proceso productivo. Por ejemplo, en la fabricación de calzado durante su etapa manufacturera en la Argentina, hasta la primera década del siglo veinte, se utilizaban pocas máquinas; la principal servía para cortar y aplanar suelas, tarea sumamente pesada y que, a la escala de producción manufacturera, hubiera demandado un descomunal gasto de mano de obra de realizarse en forma manual, por lo que fue una de las primeras máquinas que se introdujeron.

El obrero colectivo, que es el mecanismo vivo de la manufactura, está compuesto por los obreros parciales. Sus distintas funciones requieren diferentes grados de calificación de la fuerza de trabajo, por lo que se crea al interior de ésta una jerarquía que se ve reflejada en los salarios. Si no hubiera división del trabajo todos los obreros deberían contar con la fuerza y el conocimiento suficientes para realizar todas las tareas dentro del proceso de trabajo y sus salarios reflejarían este hecho. Pero, el capital paga a cada obrero un salario en función de la calificación necesaria para la operación que realiza. De esta manera, el capitalista logra reducir el costo de la fuerza de trabajo, remunerando a cada trabajador sólo por la calificación exacta que requiere para llevar a cabo su tarea. A la vez, se logra que quienes están en la cúspide de esa jerarquía y reciben los salarios más altos, no empleen tiempo (caro) en realizar actividades simples. Esto, conocido como principio de Babbage, permite disminuir la suma de salarios pagados por el empleador, aun cuando algunos obreros pocos obreros reciben salarios más altos que los que percibirían de otro modo.

En forma complementaria, al desmembrarse el trabajo en sus distintas operaciones, aparecen algunas de ellas que no requieren ningún adiestramiento previo. De esta manera, también la ausencia de calificación se convierte en una especialidad dentro de la manufactura; surge así la figura del peón, si bien su importancia se encuentra limitada en esta etapa por la preeminencia de los obreros calificados.

\section{Una maraña de formas transicionales}

La manufactura moderna se define por una progresiva mecanización de tareas, sin que éstas lleguen a conformar un sistema de máquinas. Más allá de estas proposiciones, muy poco puede afirmarse en un plano general, lo que queda son las múltiples formas en que esto se manifiesta. Existen casos sencillos, como los frigoríficos argentinos en la década del veinte en los que encontramos un proceso de mecanización periférico (TARDITTI, 1999). Sin embargo, la mayoría de los casos resultan más complejos: Marx mostró cómo, en un primer momento, al 
introducirse maquinaria era posible reproducir las condiciones de producción artesanales; en tanto este sistema no se generalizaba, era posible que un productor independiente que contaba con maquinaria, compitiese favorablemente contra manufacturas que empleaban muchos más obreros (MARX, 1982, pp. 163-6). Algo semejante ocurre en la fabricación de calzado cuando, a finales de la década del veinte, se comienza a implementar en el país el sistema de vulcanizado. Este sistema que permite pegar en forma automática el corte (la parte de arriba del calzado), con la suela y la plantilla, pronto va a dar forma a una gran industria propiamente dicha. Pese a ello, en un momento inicial este sistema favorece el desarrollo de una forma híbrida de manufactura moderna. Las primeras unidades productivas que adoptaron este sistema combinaron en sus talleres el proceso de vulcanizado, propio de la gran industria, con el trabajo manual en la mayoría de las operaciones previas. Esta combinación resultó posible porque fueron los productores más atrasados los primeros en incorporar este nuevo método, el cual les permitía con un costo relativamente reducido competir e incluso aventajar a las fábricas de mayor tamaño. Por el contrario, los grandes productores, cuyas empresas se hallaban inicialmente más mecanizadas tardaron en adoptar el nuevo sistema porque éste dejaría en desuso juegos completos de máquinas que quizás no habían sido aún amortizadas (KABAT, 2005; 2008).

El panorama resulta aún más complejo cuando la producción mecanizada, es decir la gran industria, cobra una mayor proyección en el conjunto social. En ese punto, las actividades que aún conservan una forma manufacturera pueden proveerse de insumos generados por la gran industria, que acortan los tiempos de trabajo. Las grandes cadenas de fast food, constituyen un ejemplo de este tipo de manufactura moderna, cuya productividad depende en gran medida del empleo de insumos preelaborados por la gran industria. McDonalds, por ejemplo, incluso utiliza una variedad de papas especialmente modificada genéticamente para facilitar su cocción en este tipo de locales gastronómicos (SARTELLI, 2001). Vemos aquí que opera entre distintas ramas económicas un desarrollo desigual y combinado. Estas formas de articulación particulares aparecen también en el desarrollo de diferentes ramas económicas en distintos países (SARTELLI; KABAT, 2016).

\section{La gran industria}

La gran industria se caracteriza por la aparición ya no de una máquina aislada, como puede ocurrir en la manufactura moderna, sino de un sistema de máquinas. Las máquinas no constituyen un elemento simple 
del proceso productivo, tal como ocurría durante la manufactura. En cambio, representan, una base técnica unificada. Eso se logra en un primer momento por el empleo de una misma fuerza motriz y por el mecanismo de transmisión de ésta que también, en parte, es común a todas las máquinas. El corazón de la fábrica ya no es el obrero colectivo, sino el sistema de máquinas.

Podemos diferenciar dos tipos de sistemas de máquinas: uno, producto de la combinación de máquinas homogéneas, cada una de las cuales efectúa la totalidad del proceso de trabajo. El segundo caso es el sistema de máquinas propiamente dicho, que realiza una serie conexa de procesos graduales distintos, obrados por máquinas heterogéneas, pero complementarias entre sí. En ambos casos, éste constituye un gran autómata, siempre que esté movido por la misma fuerza motriz. Encontramos una nueva variante cuando el proceso se vuelve automático. Antes de alcanzar esta etapa técnica, algunas máquinas podían requerir el concurso del obrero para realizar sus movimientos. En ocasiones, éste debía manipular ciertas partes de la máquina como si se tratara de una herramienta. Nos hayamos frente a un sistema automático, en cambio, cuando la maquinaria efectúa todos los movimientos necesarios para la elaboración de la materia prima y sólo requiere la asistencia ulterior del obrero. Un sistema automático puede a su vez perfeccionarse en ese sentido, por ejemplo, mediante la introducción de un mecanismo en la hiladora mecánica que hace que ésta se detenga al romperse un hilo. No obstante, veremos que esta tendencia a la optimización no es exclusiva de los sistemas automáticos. Bajo el predominio de la gran industria, el proceso de trabajo tiende a ser revolucionado en forma continua. Ninguna forma de división del trabajo es considerada eterna. Por el contrario, éstas son permanentemente reformuladas. Aquí vemos fusionarse tareas distintas en una sola máquina, mientras que en otros sitios se disuelven procesos anteriormente realizados juntos.

$\mathrm{Al}$ analizar el proceso productivo en sus partes componentes e intentar resolver estos pasos con el concurso de la ciencia, la gran industria tiende a reducir la diferencia que existe entre proceso productivo y proceso de trabajo. El proceso de trabajo, definido como el tiempo en el cual se añade valor al producto es parte del proceso de producción. En muchas industrias hay momentos en los que no se incorpora trabajo al producto, sino que se deja transcurrir al tiempo a la espera de que sucedan determinados procesos químicos o naturales. Estos momentos son parte del proceso productivo, pero no del proceso de trabajo. La agricultura y la producción de vinos y otras bebidas alcohólicas son casos paradigmáticos, pero muchas otras actividades tienen en menor escala estos tiempos muertos en los que no se añade valor al producto. Cuanto mayores sean 
estos tiempos muertos, más grande será también la diferencia entre proceso productivo y proceso de trabajo. La gran industria tiende a violentar estos tiempos naturales y, de esta manera, logra una mayor continuidad del proceso trabajo y aumenta la velocidad de rotación del capital. La forma en que la gran industria violenta estos tiempos naturales resulta más evidente en la producción de bienes agropecuarios, donde se han gestado importantes transformaciones en este sentido al conseguir, por ejemplo, dos cosechas anuales. En otras industrias ocurre lo mismo, aunque en forma menos dramática: en la fabricación de calzado se mojaban las distintas partes que componen los zapatos antes de adherirlas a la horma para facilitar que adquieran su forma, luego se tenía que dejar secar esos pares. En la década del treinta se introducen en la Argentina secaderos, que aceleran este proceso, reduciendo el tiempo de producción y haciendo más continuo el trabajo (KABAT, 2005; 2008).

\section{Las leyes que rigen el trabajo bajo el sistema capitalista}

Estos cambios en el proceso de trabajo han sido posibles dentro del sistema capitalista, cuya lógica los ha impulsado. Ocasionalmente, en sistemas sociales previos, pudo desarrollarse, en algunas actividades específicas y en forma eventual, la manufactura. Pero, la manufactura como forma de trabajo dominante corresponde al capitalismo, al igual que el régimen de la gran industria que es, como vimos, el régimen de trabajo específicamente capitalista, por lo que no tiene correlato en etapas históricas anteriores.

Bajo el capitalismo, el objetivo de la producción no es crear un bien, sino una mercancía que ha de venderse con una ganancia para el capitalista. Bajo el capitalismo, el trabajador es un obrero, un asalariado que percibe por su trabajo un valor inferior al que él ha creado. Esta diferencia entre el valor creado y el percibido por el obrero, la plusvalía, es apropiada por el capitalista, es su ganancia, pero sólo la obtiene tras haber vendido su mercancía en el mercado. Aquí entramos en la esfera de la circulación, de la competencia, donde el capitalista se enfrenta a otros capitalistas, el lugar donde el empresario puede realizar la plusvalía. De esto surge la unión del proceso de producción y proceso de valorización dentro del sistema capitalista, ambos regidos por la ley del valor. Se comprende, también, que el objetivo del proceso de producción y, por ende, del proceso de trabajo es la obtención de plusvalía.

Una mayor plusvalía puede obtenerse aumentando la jornada de trabajo o intensificándola (condensando más trabajo en igual tiempo) o sea aumentando el volumen de trabajo por sobre el tiempo de trabajo necesario (aquel que se requiere para que el obrero produzca valor 
equivalente al de su fuerza de trabajo -el de su reproducción). Pero esta posibilidad, empleada por si sola choca pronto con fuertes limitaciones.

En cambio, si el aumento de la plusvalía se obtiene reduciendo el tiempo de trabajo necesario, por vía de aumentar la fuerza productiva del trabajo social (igual trabajo genera más productos) el valor de los productos individuales disminuye $y$, en la medida que entran en el consumo de la clase obrera, desciende también el valor de la fuerza de trabajo, de este modo se abrevia el tiempo de trabajo necesario, aumentando la plusvalía que, en este caso, llamamos relativa. Los distintos sistemas de trabajo: cooperación simple, manufactura y gran industria son distintas formas de aumentar la fuerza productiva del trabajo, por ello se constituyen bajo el capitalismo en distintas fuentes de obtención de plusvalía relativa.

Dos tendencias se desarrollan a través de estas distintas formas: la descalificación del trabajo y a la separación entre su concepción y ejecución. Ambas se realizan de manera diferente en la manufactura y en la gran industria.

A diferencia de otros animales cuyo trabajo es instintivo y no presupone una elaboración mental, el trabajo humano implica siempre una planificación. Pero precisamente esto es lo que permite que la tarea de concebir el trabajo pueda ser separada de su ejecución. Dentro de la manufactura esta separación está dada por la división del trabajo: es el capitalista quien concibe la forma de organizar el trabajo al disponer la fragmentación de tareas. A la vez, es él quien asume la función de dirección y coordinación necesarias al convertirse el trabajo en un sistema complejo basado en la articulación de tareas parciales de distinta índole. Al extremarse la división del trabajo, esta separación entre la concepción y la ejecución del trabajo resulta más evidente. Sobre todo, cuando el capitalista comienza a contratar especialistas para el diseño de la organización del trabajo. Estos especialistas llegan a estudiar y cronometrar las distintas operaciones del obrero, subdividirlas y a indicar al operario exactamente qué movimientos ha de realizar. A su vez, esta misma división del trabajo implica una descalificación del obrero, en tanto restringe los conocimientos, pericias y habilidades que poseía.

En la gran industria, en cambio, la descalificación actúa por medio de la mecanización: el saber, la fuerza pericia o habilidad del obrero pasan a la máquina, mientras las calificaciones de los obreros tienden a igualarse en su nivel más bajo. Bajo este régimen de trabajo la separación entre concepción y ejecución se da por el desarrollo de la técnica y de la ciencia. Es el desarrollo científico lo que permite idear nuevos productos y procesos, así como la maquinaria que se utiliza en la gran industria. 
En síntesis, el proceso de trabajo bajo el capitalismo sólo se entiende si se lo interpreta como una unidad con el proceso de valorización. La necesidad de obtener más plusvalía y sobrevivir así a la competencia impulsa la transformación de la forma del trabajo. Encontramos dos tendencias que actúan bajo los distintos sistemas de trabajo: la descalificación y la separación entre concepción y ejecución del trabajo. Esta última actúa mediante la planificación de la división del trabajo durante la etapa manufacturera y a través del desarrollo de la ciencia en la gran industria. La descalificación opera por la división del trabajo durante la manufactura y mediante la mecanización el uso de la ciencia en la gran industria.

Veamos un ejemplo, en las primeras etapas de la industria gráfica, la única forma que tenía el capital de descalificar el trabajo en tareas como la composición del texto era la fragmentación del trabajo. De esa manera, aparecen tipógrafos que sólo componían el texto, otros que se ocupaban de títulos y páginas con diagramación especial y aquellos que corregían errores. Pero, con la mecanización la descalificación de los trabajadores es mucho más drástica: con la aparición de las máquinas linotipos el período de formación de los obreros experimentó una reducción radical: de los años que llevaba el proceso de formación de un tipógrafo se pasa a un entrenamiento de tan solo una semana para operar la máquina linotipo (BILL, 2007). En la Argentina el pasaje de la tipografía a las máquinas de linotipo se da en la primera década del siglo veinte. En algunas provincias del interior la tipografía subsiste por más tiempo, pero hacia la década del sesenta había prácticamente desaparecido por completo. En cambio, la composición tipográfica vía linotipo resulta dominante hasta el inicio de los años setenta. Este procedimiento había mecanizado gran parte de las tareas, pero había permitido la subsistencia de un número comparativamente reducido de tipógrafos involucrados en corrección de textos, armado de títulos y columnas. Toda esta fuerza de trabajo calificada residual fue desplazada rápidamente con los primeros avances de la composición en frío. El primer paso fue la aparición en el mercado de la IBM Composer que, entre otras tareas, justificaba automáticamente las columnas. Aun así, todavía subsistía el oficio de armador de página que consistía en el armado de las columnas, sobre mesas iluminadas desde abajo, la colocación de títulos, tirado de rayas, pegado de ilustraciones, etc., para lo que se ocupaba mucho personal. Las máquinas de composición en frío sobre papel fotográfico superaron a la IBM Composer en variedad de tipos, la calidad final y la posibilidad de armar las columnas con títulos y gráficos, con lo que se eliminaba el oficio de armador de página. Este proceso luego se profundizó con el empleo de computadoras. La simplificación de los procesos de impresión permitió un proceso de 
integración de diferentes especialidades de la rama gráfica que pasaron a integrarse en un mismo taller (KABAT et al., 2004). La rama gráfica es un ejemplo de la innovación permanente y de la descalificación generada bajo el régimen de gran industria.

En un artículo anterior hemos analizado los cambios recientes en la organización del trabajo en la producción de cereales y su relación con la expansión del contratismo rural. Aquí nos interesa recuperar algunos elementos de ese artículo útiles para pensar el problema de las calificaciones y el control en el régimen de gran industria.

La siembra directa comienza a aplicarse en la Argentina a inicios de los ochenta. Este método elimina el laboreo de la tierra (la remoción de malezas y residuos vegetales de la cosecha anterior) y también la tarea de arar. Con la siembra directa no se desmaleza la tierra ni se abren surcos. Con maquinaria específicamente diseñada para eso se deposita la semilla exactamente a la profundidad requerida. Al sembrar sin remover los rastrojos anteriores se incrementa el crecimiento de malezas. Por eso es importante el desarrollo de mecanismos para su control de un modo eficiente y económico. Esto se logra con el uso simultáneo de herbicidas especiales y semillas transgénicas diseñadas para resistir estos agroquímicos.

El uso de sistemas computarizados, el monitor de rendimiento y el sistema de guía por GPS permiten emplear en un lote dosis diferenciadas de semilla, fertilizantes y pesticidas, en vez de aplicar cantidades conformes al promedio de la superficie de la unidad productiva.

La maquinaria agrícola ha tendido también a automatizarse. Ello ha eliminado tareas secundarias como la del obrero que controlaba que la semilla cayera correctamente, ahora remplazado por un sensor colocado en las cosechadoras. La siembra directa ha prescindido de cuatro de cada cinco empleos anteriormente demandados por la labranza tradicional.

Las tareas se simplifican y requieren menos saberes y experiencia. Como en otras ramas, mucho se habla de las supuestas nuevas calificaciones del trabajador agrícola, pues se confunde y asimila la complejidad técnica de la maquinaria con la de los saberes del obrero que la opera. Por el contrario, la automatización de la maquinaria simplifica el trabajo que no requiere un aprendizaje especial por parte de los maquinistas. Tal como señala el Manual de prevención de riesgos rurales de la Superintendencia de riesgos de Trabajo, en general, la maquinaria agrícola se emplea tras una breve explicación oral (SARTELLI; KABAT, 2009). Este hecho fue confirmado por el gerente Nacional para el área Rural de Manpower, Argentina a principal empresa de contratación de empleos en el medio agrario. Ante nuestra pregunta de si era cierto que la nueva maquinaria demandaba altas calificaciones, nos respondió: 
Es una mentira... El que la programa seguramente no va a ser el que se suba arriba de la máquina. Le dicen que comando tiene que tocar, tené cuidado si se prende la luz roja y dale para delante cuando esté la luz verde prendida. (...) Tu única preocupación es tener gasoil. (ROSSIN, 2008)

Los salarios de los operadores de estas máquinas rurales son muy bajos y no resultan superiores al de los obreros rurales manuales. Con la agricultura de precisión también se simplifica el control del trabajo. La misma máquina guarda registro informático de las tareas realizadas, que pueden ser fácilmente verificadas. A su vez, si los gerentes de la explotación desean un mayor control en el momento mismo que se realiza la siembra o cosecha basta con que empleen un segundo GPS para la supervisión (SARTELLI, 2008, pp. 75-79).

A su vez, todas las instancias de este proceso son permanentemente modificadas con el concurso de la ciencia. En los últimos años, se inició el empleo de drones con cámaras multiespectrales para la planificación y monitoreo de cultivos. La mayoría de los establecimientos agrarios no comparaban estos equipos, sino que contrataban el servicio de compañías que realizaban la tarea. Para lo cual, un empleado se dirigía a cada campo, operaba el dron y luego procesaba y suministraba la información. Según un informante, quienes se dedicaron a esta tarea, sabían que la misma sólo sería viable por un plazo máximo de cinco años, porque ya entonces resultaba previsible que los drones fueran desplazados por los nanosatélites. Estos últimos ofrecen un mejor servicio (hasta 3 imágenes diarias), con información sobre humedad de los suelos a mayor profundidad, sin que un operario tenga que desplazarse al terreno (INFORMANTE ANÓNIMO, 2018). Efectivamente, se considera que los nanosatélites en breve terminarán de desplazar el uso de drones de monitoreo. Pero, los drones comienzan a tener nuevas funciones de acción directa como pulverización, siembra o poda. Es decir, los drones constituyen piezas claves de la denominada robotización de las tareas rurales. Se espera que esto genere una mayor coordinación de los distintos equipos empleados. La comunicación de las actividades y sus parámetros, puede ser enviada en forma continua a la PC de la oficina o a un centro de operaciones en tiempo real, donde se toman las decisiones y se le envía a cada robot (dron) la tarea a ejecutar (MARINELLI, 2018).

Vemos aquí en el agro un desarrollo común a otras áreas que es el rol que juegan los sistemas computarizados y medios de comunicación asociados en dotar de unidad al sistema de máquinas empleadas. Mientras que en el siglo XIX y gran parte del siglo XX la base técnica común de un sistema de máquinas estaba dado sólo por el uso de una energía en común (misma fuerza motriz y sistema de distribución), el uso de un sistema operativo común que permite centralizar comandos y control de las tareas 
brinda mayor homogeneidad a sistemas de máquinas, incluyo en aquellos casos hasta entonces poco articulados por la distribución espacial de las tareas.

\section{Braverman y la administración científica del trabajo}

Pese a la potencialidad de las nociones elaboradas por Marx para estudiar los procesos de trabajo, muchos marxistas las ignoran y emplean, en cambio, otras nociones cuya coherencia con el marco teórico marxista en general no cuestionan. Este es el caso de Harry Braverman (BRAVERMAN, 1988). En esta sección intentamos mostrar los deslizamientos y contradicciones en los que incurre Braverman a partir del concepto de taylorismo. A la vez, sostenemos que la tesis básica de Braverman, el deskilling, es correcta, pero que no necesita ser explicada a partir de la categoría de taylorismo, sino que se entiende mejor desde la conceptualización marxista clásica, tal como hemos intentado demostrar al hablar de las leyes del trabajo bajo el capitalismo.

Braverman considera que su obra es una continuación, una descripción pormenorizada y contemporánea de un proceso que Marx ya había analizado en sus líneas fundamentales. Su trabajo generó una de las polémicas más fructíferas del campo marxista (SMITH, 1996; CUNHA, 2014; LITTLER, 1990). Las críticas más fuertes se dirigieron hacia una de sus tesis centrales, la tendencia capitalista a reducir la calificación requerida en la fuerza de trabajo, el “deskilling”. En este caso, sus críticos hacen referencia a nuevas ocupaciones que requieren trabajadores altamente calificados. A nuestro juicio, esto no invalida la tesis de Braverman, ya que pueden surgir nuevas actividades, pero éstas probablemente recorran el camino que las demás han seguido, algo de lo que hay evidente prueba actual. En otros términos, el hecho de que en sectores económicos nuevos se demande fuerza de trabajo calificada no contradice la tendencia al deskilling.

Sin embargo, hay otros aspectos centrales de esa gran obra que pueden ser cuestionados: estos son la generalización para todo el capitalismo de tendencias propias de la manufactura y una aparente perpetuidad del obrero parcelario.

Braverman, siguiendo a Taylor, enfatiza que el taylorismo puede aplicarse sobre cualquier base técnica dentro del sistema capitalista (BRAVERMAN, 1988, p. 106). Resulta evidente que, si una forma de trabajo es aplicable a cualquier etapa técnica, la misma resulta poco útil para historizar los procesos de trabajo. En ese sentido, la noción de taylorismo significa un retroceso frente a las categorías marxistas tradicionales. 
Pese a la imprecisión y ambigüedad del término taylorismo, consideramos que sus rasgos centrales corresponden a las características de la etapa manufacturera. Cuando Braverman analiza ejemplos que corresponden a formas de gran industria, tiende a resaltar los fenómenos ligados a la perpetuación de características manufactureras que, tal como lo señala Marx, vegetan durante un tiempo en el nuevo régimen. De esta manera, se enfatizan las continuidades por sobre las rupturas, efecto que se refuerza por su falta de atención hacia los rasgos novedosos que la gran industria trae aparejados: tanto los cambios en la división del trabajo como la aparición de una estructura productiva completamente objetiva son desatendidos. De este modo, las características centrales, específicas de la gran industria, no son contempladas; de ahí surge la dificultad que existe para pensar, desde el modelo de Braverman, los fenómenos que representan una profundización del régimen de gran industria y la ruptura con elementos remanentes del régimen manufacturero, como lo sería la polifuncionalidad.

Antes de avanzar debemos recordar las características que asume la división del trabajo bajo la manufactura y bajo la gran industria. En la manufactura, las diversas operaciones manuales, producto de la fragmentación del oficio, requerían fuerza, pericia o habilidad; una vez que el obrero desarrollaba esas aptitudes se veía confinado de por vida a esa tarea en la que se había especializado. En cambio, en la gran industria, basada en el empleo de maquinaria, no se requiere mayor fuerza o pericia de parte de los obreros, por lo que ya no resulta necesario establecer este tipo de especializaciones. Naturalmente, distintos obreros operan distintas máquinas, pero no hay ninguna necesidad de que operen siempre la misma máquina porque estas actividades no requieren habilidades específicas. La división sexual del trabajo de esta manera pierde en la gran industria su fundamento técnico. La gran industria al objetivar el trabajo, destruir el sistema de aprendizaje de oficio y eliminar los requisitos especiales de fuerza física, anula la base de la anterior división genérica sexual del trabajo. La gran industria establece los cimientos para el desarrollo de nuevas relaciones de género, tanto en la fábrica como en el ámbito familiar. En palabras de Marx:

Ahora bien, por terrible y repugnante que parezca la disolución del viejo régimen familiar dentro del sistema capitalista, no deja de ser cierto que la gran industria, al asignar a las mujeres, los adolescentes y los niños de uno u otro sexo, fuera de la esfera doméstica un papel decisivo en los procesos socialmente organizados de la producción, crea el nuevo fundamento 
económico en que descansará una forma superior de la familia. (MARX, 1999, t. 1, v. 2, p. 596).4

Otras diferencias oponen la división de trabajo en la manufactura y en la gran industria. En la primera el trabajo se divide en forma sistemática, pero siempre teniendo en cuenta las capacidades del hombre, en cambio la división de tareas en la gran industria se establece sin tomar en cuenta la mano humana. En la manufactura

Si bien el obrero ha quedado incorporado al proceso, también es cierto que previamente el proceso ha tenido que adaptarse al obrero. En la producción fundada en la maquinaria queda suprimido este principio subjetivo de la división del trabajo... (MARX, 1999, t. 1, v. 2, p. 462)

En la manufactura existe un principio subjetivo sobre el que se estructura la división del trabajo: se reparten las distintas actividades entre los obreros de acuerdo a sus capacidades. De ahí la importancia que adquiere la correcta selección del personal.

En la gran industria, que carece de este principio subjetivo, esto funciona al revés: son los obreros quienes son distribuidos entre las máquinas. Esto último es posible merced a la existencia de una base objetiva sobre la cual es posible asignar distintos trabajos a los obreros sin tomar en cuenta su capacidad. Esta base objetiva está dada por el sistema de máquinas.

Aquí volvemos sobre algunos puntos adelantados al referirnos al gran autómata: bajo el predominio de la gran industria, el proceso de trabajo tiende a ser revolucionado en forma continua. Ninguna forma de división del trabajo es considerada eterna, en cambio éstas son permanentemente reformuladas. Así vemos fusionarse tareas distintas en una sola máquina, mientras que en otros sitios se disuelven procesos anteriormente realizados en forma conjunta. Recordemos el carácter revolucionario de la base técnica propia de la gran industria. La gran industria no sólo no requiere la existencia de obreros especializados, confinados en forma permanente a una misma tarea, sino que, además, la revolución continua del proceso productivo vuelve necesario el cambio de trabajo, la mayor polifuncionalidad posible de los obreros.

La división del trabajo bajo el taylorismo corresponde a los rasgos propios de la manufactura. Los componentes centrales de la manufactura, la división del trabajo y el obrero parcelario son conducidos a su extremo por el taylorismo. El propio Taylor gustaba de llamar a su sistema "la moderna subdivisión del trabajo". Esta definición era atinada, pues la organización científica del trabajo significó, entre otras cosas, la

4 En este sentido resulta correcta y sumamente pertinente la relación que establece Moraes Neto entre la división manufacturera del trabajo y el empleo de mujeres orientales en cadenas de montaje (MORAES NETO, 1991, p. 98). 
descomposición en operaciones extremadamente simples de las tareas en las que se había dividido anteriormente el proceso de trabajo.

En la primera forma de la división del trabajo, el capitalista desbarata los oficios y los devuelve en migajas, en forma tal que el proceso en su conjunto no es ya el dominio de ningún obrero en particular. Luego, como lo hemos visto, el capitalista realiza un análisis de cada una de las tareas distribuidas entre los obreros, con un ojo puesto en las operaciones individuales. Es en la época de la revolución científico-técnica cuando el patrón se plantea el problema de dominar el proceso como un todo y controlar cada uno de sus elementos sin excepción. (BRAVERMAN, 1988, pp. 201-2)

Si bien este segundo paso requiere, como elemento novedoso, todo el despliegue de la administración patronal que Braverman describe en forma pormenorizada, su esencia sigue siendo la división del trabajo y la asignación permanente de cada una de las partes resultantes al obrero individual. Opera aquí el mismo principio que es llevado por el taylorismo hasta sus últimas consecuencias; en ese sentido, éste puede ser visto como el punto más alto del régimen manufacturero.

Lo mismo ocurre con el principio de Babbage, que corresponde a la división manufacturera del trabajo. Braverman considera que este principio es un rasgo fundamental de todo proceso de trabajo capitalista (BRAVERMAN, 1988, p. 103). Pero, la jerarquía de calificaciones y salarios de los obreros, propia de los obreros manufactureros tiene su fundamento en la especialización. La misma constituye un producto de la necesidad de preservación de las habilidades adquiridas por un obrero al realizar manualmente y en forma vitalicia una tarea parcial. Con la gran industria el fundamento de esta jerarquía desaparece, pues ésta no demanda diferentes niveles de fuerza y pericia. Estos han sido concentrados en la máquina. La tendencia central en esta nueva etapa es a la igualación de los conocimientos, aunque en su grado más bajo.

Con la herramienta de trabajo, se transfiere también del obrero a la máquina el virtuosismo en el manejo de aquella. (...) Queda abolido, con ello, el fundamento técnico sobre el que descansa la división del trabajo en la manufactura. Por eso, en lugar de la jerarquía de los obreros especializados, característica de esa división del trabajo, aparece en la fábrica automática la tendencia a la equiparación o nivelación de los trabajos que deben ejecutar los auxiliares de la maquinaria... (MARX, 1999, t. 1, v. 2, p. 512).

Como el sistema de máquinas no es un mecanismo perfecto desde sus inicios, eventualmente determinadas máquinas requieren mucha habilidad del obrero para operarlas, y permiten que se mantenga parte de la jerarquía de calificaciones propia de la manufactura. Pero en esos casos, muy pronto el capital perfeccionará la maquinaria para socavar esa pericia. 
Por lo tanto, a medida que la gran industria se desarrolla, la ley de Babbage encuentra un campo de acción menor.

En síntesis, la división del trabajo, la creación del obrero parcelario y la puesta en práctica del principio de Babbage, constituyen elementos típicos de la manufactura, los cuáles son desarrollados por el taylorismo hasta el extremo de sus posibilidades. El taylorismo es, entonces, la máxima expresión de la manufactura. Si encontramos que éste se aplica en sistemas que han superado esta etapa, mediante la introducción medianamente generalizada de maquinaria, éstos son, en general, casos de manufacturas modernas, o bien de gran industria no automatizada, especialmente aquellas donde la operación de las máquinas requiere todavía un grado elevado de calificación del obrero.

A estas mismas conclusiones nos conduce el estudio de las preocupaciones propias del taylorismo. Es interesante ver cómo éstas se concentran en superar los problemas propios de la manufactura: enajenar la pericia de los obreros y disminuir la importancia del factor subjetivo en la producción. Estos problemas, que desaparecen en la gran industria, demandan al capital gigantescas y renovadas energías, cuando éste aún no ha alcanzado el nivel técnico de aquella. De ahí todos los esfuerzos empresariales para lograr el control del proceso de trabajo, en los que Braverman pone tanto énfasis.5Vemos como, bajo el taylorismo se intenta denodadamente, a través de la administración técnica-gerencial, lo que la mecanización del proceso productivo, y más aún su automatización, lograrían fácilmente. Tanto en las motivaciones de Taylor como en los en los principios establecidos por él y analizados por Braverman, puede hallarse el sentido que subyace a todos estos afanes:

El impulso para las primeras investigaciones de Taylor provino de su alarma ante la certidumbre de que los obreros sabían más sobre su trabajo que la gerencia. Taylor explica, incluso, que aunque los capataces hubieran sido ellos mismos excelentes obreros, su conocimiento no era sino una ínfima parte del que poseían en forma conjunta los trabajadores. Es fácil comprender que este conocimiento combinado de los obreros, muy superior al que los capataces y gerentes podrían reunir, es el del obrero colectivo, que constituye, al decir de Marx, el órgano vivo de la

5 Es llamativa la preeminencia que Braverman brinda a las estrategias gerenciales destinadas a lograr el control sobre el trabajo, incluso a costa de desatender el modo en que los cambios objetivos del proceso productivo modifican esas necesidades de control. Por esto es posible sostener que su obra contempla la lucha de clases mucho más de lo que generalmente se reconoce. Se podría decir que, desde este ángulo, su trabajo es la contrapartida del de Montgomery; ambos se centran en las luchas por el control, pero mientras uno analiza las estrategias de la burguesía, el otro estudia a la clase obrera (MONTGOMERY, 1988). 
manufactura. ${ }^{6}$ Todos los preceptos de la management científico están dirigidos contra este hecho.

El primer principio puede ser llamado disociación del proceso de trabajo de la pericia de los obreros. El proceso de trabajo debe mantenerse independiente del oficio, de la tradición y del conocimiento de los obreros. Lo que es más, no debe depender para nada de las capacidades de los obreros, sino enteramente de las prácticas de la gerencia. (BRAVERMAN, 1988, p. 139)

Si nos encontráramos frente a un régimen de gran industria, sería innecesario el desarrollo de toda una estructura gerencial para disociar el proceso de trabajo de la pericia del obrero, puesto que la existencia de un sistema de máquinas invalidaría de por sí esa pericia, por eso Marx habla de subsunción real, frente a la subsunción formal propia de la manufactura. Lo mismo ocurre con los restantes principios taylorianos que Braverman analiza (BRAVERMAN, 1988, pp. 139-48).

El segundo establece la necesidad de remover del taller el trabajo cerebral y concentrarlo en la gerencia, o sea, disociar la concepción y la ejecución del trabajo. Por último, el tercero indica el uso del conocimiento del proceso de trabajo reunido por la gerencia para controlar cada paso de éste a través de la especificación de las tareas, indicando lo que debe hacerse, cómo y en qué tiempo.

En la gran industria ya se ha consumado el divorcio entre la concepción y la ejecución del trabajo. Esto se manifiesta, fundamentalmente, en el lugar ocupado por la ciencia en la configuración del proceso productivo a partir del diseño de la maquinaria.7Esta separación, pues, ya está dada de antemano en el régimen de gran industria y posteriormente, no demanda mayores esfuerzos de parte de la gerencia. Del mismo modo, el ritmo de trabajo, así como las operaciones que ha de realizar el obrero están regidos por las máquinas y sus movimientos, a los que el obrero debe adaptarse. En este contexto sería superflua la tarjeta escrita de instrucción o cualquier otra forma de especificar la tarea; con mayor razón aún, si nos encontráramos ante un sistema de máquinas automático.

A través de sus técnicas de control, la gerencia aspira, según Braverman, al ideal, nunca alcanzado de desplazar al trabajo como elemento subjetivo dentro del proceso productivo. La gerencia aparecería, así como el único factor de subjetividad, al tiempo que lograría subordinar

6 Comúnmente se denuncia la descalificación del trabajo artesanal frente al manufacturero, olvidando que, si bien la división de tareas empobrece los conocimientos de los obreros individuales, no ocurre lo mismo al obrero colectivo. En cambio, en la gran industria, el obrero colectivo es descalificado.

7 En cambio, Braverman al estudiar la ciencia, privilegia lo que él denomina revolución gerencial. 
al trabajo, transformándolo en un elemento objetivo (BRAVERMAN, 1988, pp. 202-3).

En síntesis, la gerencia intenta a través de la subdivisión del trabajo, la concentración del conocimiento y la especificación de tareas con tiempos, pautas y movimientos establecidos, substraer los aspectos subjetivos del trabajo del control de los obreros, pero, como veremos, esto no equivale a removerlos del proceso de trabajo.

Esta misma necesidad y los gigantescos esfuerzos de la gerencia para resolverla se relacionan con la inexistencia de una estructura objetiva del proceso productivo al margen de los obreros, o sea, la ausencia de un sistema de máquinas y, por lo tanto, de gran industria. Vemos aquí, nuevamente, cómo los problemas que el taylorismo intenta resolver son acuciantes para la etapa manufacturera e irrelevantes para la gran industria. Frente a este problema, el taylorismo representa una vez más la respuesta más avanzada que la manufactura puede brindar: intenta, por medio del estudio de los movimientos del obrero especificar sus tareas, predeterminando la forma y el tiempo de completar el trabajo, pero al hacer esto, choca con sus propios límites. Porque, aun cuando todas las tareas fuesen especificadas y estas directivas pudieran ser cumplidas exactamente por los obreros en todos sus detalles, el elemento subjetivo del trabajo no se habría eliminado: si bien el obrero se adapta a esas indicaciones y a la forma en que la gerencia decide subdividir el trabajo, antes ésta debe estudiar las capacidades de los obreros, sus movimientos en el trabajo, hasta su desgaste por cansancio físico o psicológico. Recién entonces se halla en condiciones de reorganizar el proceso de trabajo, pero sobre la base de los conocimientos que ha recabado sobre este elemento subjetivo. Marx explica cómo esto cambiará con el pasaje del régimen de manufactura al de gran industria, que suprime la base subjetiva de la división manufacturera del trabajo.

Hasta qué punto el taylorismo queda anclado dentro de estos límites propios de la manufactura y, a la vez, desarrolla todas las potencialidades latentes en ella, lo demuestra la cantidad de tiempo, personal, y recursos técnicos empleados para estudiar los movimientos de los obreros: se desarrolla incluso toda una rama de la ciencia, la fisiología del trabajo, cuyo desarrollo se halla muy ligado al taylorismo y tenía en el estudio de la fatiga uno de sus campos privilegiados de estudio. Como señala Benedito Rodriguez de Moraes Neto, el conocimiento científico bajo el taylorismo es apenas un soporte para que el capital explore las particularidades del hombre y, por otro, que perfecciones los mecanismos de control del obrero colectivo (MORAES NETO, 1989, p. 41).

Mientras Braverman describe magistralmente la forma en que el taylorismo lleva a un extremo la división manufacturera del trabajo, no 
alcanza a vislumbrar los cambios que el régimen de gran industria impulsa en este renglón. Al centrarse en la descripción del taylorismo, donde las máquinas juegan un rol secundario, Braverman tiende a subestimar su papel en períodos posteriores. Cuando analiza el rol de la ciencia, pareciera valorar la administración científica como uno de sus mayores logros, restando a la creación de maquinaria parte de su importancia en esto. $\mathrm{Su}$ énfasis en la apropiación por parte de la gerencia de los conocimientos del obrero, le impide ver que no todos los conocimientos científicos se obtienen de este modo. En algunos casos, la división del trabajo facilita su posterior mecanización, pero en otros, ya bajo la gran industria, el proceso productivo es completamente reelaborado. En estos casos, que representan la tendencia dominante, no se parte del conocimiento que posee el obrero, sino del desarrollo autónomo de la ciencia. Un ejemplo de esto es la aparición del proceso de vulcanizado en las fábricas de calzado: gracias al empleo de nuevos materiales, entre ellos el caucho, mediante un proceso químico y con máquinas automáticas, se pegan en forma automática las partes del calzado que antes eran cosidas a través de cincuenta operaciones. Del mismo modo, el desarrollo actual de los nanosatélites no se basa en la expropiación de saberes previos de los obreros rurales, sino que constituye un desarrollo autónomo basado en la ciencia. Este tipo de avances se genera al margen de los conocimientos de los obreros, saberes que, sin embargo, termina por volver obsoletos. Naturalmente, el desarrollo de la ciencia requiere la aparición de una capa obrera dedicada a este desarrollo y aplicaciones prácticas del conocimiento. Pero, la aparición de esta nueva fracción obrera, no contradice la tendencia general al deskilling, ya que esta fracción menor es la que posibilita la descalificación masiva de tareas.

Aquí volvemos a nuestra tesis acerca de los componentes del taylorismo; Braverman define el taylorismo a partir de dos tendencias centrales: división del trabajo y descalificación. Ya hemos planteado que se debe distinguir ambas tendencias: la descalificación es, ciertamente, una tendencia general del capitalismo. En esto Braverman acierta y su análisis en este aspecto es brillante; tanto cuando describe la descalificación en etapas del trabajo parcelario, fundamentalmente manual, como cuando describe la pérdida de conocimiento que implican los diferentes grados de automatización. En cambio, la tesis sobre la división del trabajo y la creación del obrero parcelario, es esencialmente válida para el período manufacturero, no así para la gran industria: Braverman describe certeramente cómo opera esta tendencia antes de la aparición de la gran industria, logrando avances importantes por dos caminos diferentes. Por un lado, gracias a su análisis de la parcelación del trabajo bajo el taylorismo, es posible comprender, a nuestro juicio, cómo éste acentúa esa 
tendencia propia de la manufactura, llevándola a su máximo. En segundo lugar, Braverman describe con singular lucidez como la manufactura (que él estudia bajo la forma del taylorismo), se abre paso en nuevas actividades, por ejemplo, cuando en el capítulo quince de la obra que analizamos demuestra cómo se divide el trabajo de oficina. Pero, precisamente, porque esta tendencia corresponde a la manufactura y no a la gran industria, encuentra dificultades al trasladarla a los sectores económicos donde predomina el sistema fabril. En este ámbito, su análisis se vuelve más unilateral: no da cuenta del carácter prescindible que cualquier forma de división del trabajo adopta frente a la gran industria y tampoco puede explicar cómo, en ocasiones, ésta toma una dirección contraria a la que él supone que le es propia. En este terreno, su enfoque se vuelve parcial; tiende a desconocer los fenómenos nuevos, mientras utiliza ejemplos que pueden ser considerados casos transicionales, o rémoras del sistema manufacturero para probar el funcionamiento pleno, durante todo el capitalismo, del principio de Babbage como tendencia dominante. Por el contrario, se puede observar que, a medida que el régimen de gran industria se profundiza y los sistemas de máquinas se vuelven más continuos a la par que se perfeccionan, la división del trabajo y el principio de Babbage pierden relevancia en la determinación del deskilling. Si el principio de Babbage continúa actuando en algunos sectores de la gran industria lo hace en forma secundaria, frente a la ley que tiende a equiparar en el nivel más bajo todas las calificaciones. En un primer momento, la descalificación se produce por la división del trabajo; mientras que en la etapa propia de la gran industria ésta opera a través de un medio aún más poderoso, la objetivación del proceso de trabajo.

\section{El fordismo desde el ángulo marxista}

El concepto de fordismo, al igual que ocurría con el de taylorismo, no puede asociarse a una etapa determinada en la organización de trabajo. Pero existe una diferencia: su elemento central, la cadena de montaje, ${ }^{8}$ no existía en el momento en que Marx formuló su análisis sobre el trabajo. Sin embargo, su función dentro del proceso de trabajo puede deducirse de su obra. En la manufactura, las diversas operaciones del trabajo artesanal son, disociadas, aisladas y yuxtapuestas en el espacio. El aislamiento de las tareas es algo inherente a la misma base técnica de la producción, pero esta incomunicación de sus distintas fases trae consecuencias costosas al capital:

${ }^{8}$ La definición del fordismo a partir de la cadena de montaje es una característica de los autores regulacionistas (AGLIETTA, 1988, pp. 95-6). 
Para establecer y conservar el nexo entre las funciones aisladas, se vuelve imprescindible transportar continuamente el artículo de unas manos a otras y de un proceso a otro. Desde el punto de vista de la gran industria, se presenta esto como una limitación característica, costosa e inmanente al principio de la manufactura. (MARX, 1999, t. 1, v. 2, p. 419)

Tempranamente se desarrollaron diversos mecanismos para transportar los artículos de una sección a otra y, de esta manera, subsanar en parte, aunque sin resolver, los problemas que presenta el aislamiento de las fases productivas. Así aparecieron los sistemas de rieles con ganchos en los frigoríficos o los carritos para trasladar los zapatos en la industria del calzado. La cadena de montaje no es más que una nueva forma de estos artefactos, sólo que mecanizada y, por eso mismo, la más efectiva de ellas. En su forma original, tal como aparece en la producción de autos, la cadena de montaje aligera el problema de la división manufacturera del trabajo y el correspondiente aislamiento de las secciones, pero no lo resuelve en tanto no modifica la base sobre la que se asienta esa forma de división del trabajo. Esta llegará a cambiar sólo cuando el medio de trabajo, el instrumento que opera sobre la materia prima, se modifique. Cuando este paso sea dado, la cadena de montaje realizará la misma labor que antes, pero transportando el material de unas máquinas a otras.

En resumen, la cadena de montaje surge para subsanar un problema originado en la manufactura a partir del aislamiento de tareas. En su forma clásica el fordismo es una manufactura moderna pues la cadena de montaje representa un elemento de mecanización periférica (de una tarea accesoria, no central) dentro de un proceso de trabajo fundado esencialmente en el trabajo manual (BRAVERMAN, 1988, p. 229).

Lo planteado aquí coincide en esencia con lo señalado por Benedito Moraes Neto, cuando señala que el fordismo busca resolver un problema inmanente de la producción manufacturera cuando intenta "trazer o trabalho ao operário em vez de levar o operário ao trabalho" (MORAES NETO, 1991, p. 52).

\section{Gran industria y después... (algunas consideraciones finales)}

Una de las objeciones que ha recibido Braverman, además de las ya mencionadas, ha sido la de no tomar el lugar de trabajo como un espacio de lucha (BURAWOY, 1989; MONTGOMERY, 1985). Esta misma crítica es frecuentemente reproducida frente a los estudios sobre proceso trabajo realizados por investigadores del CEICS a partir de las nociones marxistas de manufactura y gran industria; aunque en estos casos sólo en forma excepcional la misma se expresa en publicaciones escritas o en debates públicos, como ejemplo de estos casos pocos frecuentes puedes verse 
SCHVARZER (2006) y PELAEZ (2017). Es significativo que este tipo de argumentación se sostenga contra una línea de investigación que ha estudiado conflictos obreros vinculados con la organización del trabajo (BIL, 2016 y 2018; HARARI, 2014; KABAT; HARARI, 2014). Pero, esto mismo muestra que la cuestión en debate no es si se contempla o no esta dimensión de análisis, es decir, la lucha de clases, sino la jerarquía explicativa de ambos niveles de análisis. Los críticos politicistas cuestionan que no se considere a la lucha de clases como la principal fuerza determinante detrás de las transformaciones de los procesos de trabajo.

En base a esta crítica ha surgido una corriente al interior del marxismo que privilegia el estudio de las luchas obreras como motor de los cambios en los procesos de trabajo. David Montgomery, bajo este supuesto, intenta demostrar que los obreros resistieron e incluso vencieron momentáneamente al movimiento por la organización científica del trabajo en Estados Unidos. Así, sostiene que la organización del trabajo no responde a un determinismo tecnológico, sino a la lucha de clases (MONTGOMERY, 1985). Este presupuesto niega la existencia de legalidad alguna en los cambios de la organización del trabajo. Sin embargo, el paso a la manufactura primero y a la gran industria después ha sido el camino obligado que transitaron y transitan todos los sectores económicos en los distintos países, más allá de los zigzagueos coyunturales que la lucha de clases les imprime (KABAT, 2005; BILL, 2007; HARARI, 2015). Esta concepción que ignora las legalidades históricas de las transformaciones de los procesos de trabajo hace, por eso mismo, una apología del obrero artesanal o manufacturero. 9

Es llamativo que un autor que ha desarrollado ampliamente esta línea subjetivista de los procesos de trabajo, como Paul Thompson, termine señalando la creciente separación existente entre estudios del proceso de trabajo y economía política como un problema a ser superado (THOMPSON, 2010). Esa vinculación solo puede establecerse si los estudios del proceso de trabajo recuperan su eje en aspectos objetivos y recobran una perspectiva histórica amplia. Esto último implica el reconocimiento de la complejidad de procesos históricos reales, con su particular desarrollo en distintas ramas económicas y en espacios nacionales específicos. A nuestro juicio, esta perspectiva es de especial interés para los países latinoamericanos, puesto que los estudios de los procesos de trabajo permiten un abordaje superador de las trayectorias de

\footnotetext{
9 Hemos cuestionado esta mirada nostálgica de los obreros manufactureros, porque sobrevalora la capacidad de autocontrol del trabajo que estos tenían y porque ignora el carácter corporativo, y en ese sentido reaccionario del obrero de oficio (SARTELLI; KABAT, 2014). Encontramos cierto paralelismo entre esta crítica y el cuestionamiento formulado por Benedito Moraes Neto a Gorz (MORAES NETO, 1991, pp. 120-3).
} 
estas economías tanto frente de las explicaciones liberales como de las dependentistas (SARTELLI; KABAT, 2016).

El estudio del conjunto de las ramas económicas es crucial para poder caracterizar el régimen de trabajo actual del capitalismo y no confundir el estancamiento de una rama, que puede deberse a factores específicos del sector, con el estancamiento técnico del conjunto de la producción capitalista. En gran medida, los errores de Braverman se fundan en su adscripción a la tesis del capital monopolista (SARTELLI; KABAT, 2014; ROWLINSON; HASSARD, 1994). A partir de este marco teórico imagina la aparición de un nuevo régimen de trabajo, el taylorismo conformado por la combinación de tendencias manufactureras y de la gran industria. En realidad, él observa el desarrollo de la manufactura en unas ramas y de la gran industria en otras. Braverman posee un agudo conocimiento empírico de los procesos de trabajo, pero falla en su conceptualización.

La obra de Benedito Moraes Neto presenta, a nuestro juicio, valiosos elementos para entender las mutaciones de los procesos de trabajo con una mirada muy aguda en el análisis crítico tanto de la obra de Braverman como de los autores regulacionistas. Sin que esto socave en nada lo anterior, tenemos un matiz interpretavivo en cierto punto diferente respecto del rol histórico del taylorismo-fordismo. Compartimos la idea de que, en tanto formas de trabajo manufactureras, no representan la vanguardia de la organización de trabajo capitalista. Pero, a nuestro juicio, esto no quiere decir que su emergencia implique la reproducción de una etapa del trabajo ya superada históricamente (MORAES NETO, 1991, p. 59). A nuestro juicio, al no ser el proceso de trabajo una actividad unilateral del hombre, sino, como planteamos en la introducción, el resultado de su interacción con la naturaleza, en diversas ramas los obstáculos a la transformación del proceso de trabajo son muy disímiles y, por tanto, no es posible considerar, ni siquiera hoy, a la manufactura como una etapa de trabajo superada en forma absoluta en todas las actividades humanas. No creemos tampoco que pueda hablarse de un "congelamiento" de la forma taylorista por más de 50 años en la industria automotriz (MORAES NETO, 1991, p. 59). Si se mira la industria automotriz en más detalle se observa cómo algunos sectores de la misma van mecanizándose progresivamente. De hecho, cómo explica Harari en este mismo volumen (HARARI, 2019) el autopartismo avanza más rápido que las terminales automotrices hacia la gran industria. Si uno mira por fuera de la industria automotriz, la década del sesenta presenta avances importantes de la mecanización en otras ramas, como ocurre, por ejemplo, en la industria gráfica arriba mencionada. En ese sentido, no nos parece correcto hablar del fordismo como "desvío mediocrizante" (MORAES NETO, 1991, p. 59). 
Tal caracterización sólo tendría sentido si todas las industrias recorrieran las transformaciones de los procesos de trabajo a un mismo ritmo. Pero, esto no es posible tanto por la existencia de diversos grados de resistencia que la naturaleza opone a su transformación por el hombre, como por la aparición de nuevas actividades antes inexistentes. En ese mismo sentido, resulta correcta la apreciación de que los conflictos laborales asociados por la bibliografía a una resistencia al talylorismo-fordismo, representan una nueva manifestación de la rebelión típica del obrero manufacturero (SARTELLI; KABAT, 2014; HARARI, 2019; MORAES NETO, 1991, pp. 54; 58 ), lo que no quiere decir que el taylorismo reabriera un frente de combate ya clausurado históricamente por el capital (MORAES NETO, 1991, pp. 54; 58). Esta disputa estaba cerrada en ciertas ramas económicas, lo que no implica que necesariamente estuviera clausurada en todas las ramas de la economía. De hecho, en la actualidad podemos encontrar signos de resistencia a los cambios de la organización del trabajo entre médicos, docentes y otros profesionales, afectados por una creciente división del trabajo y una muy embrionaria mecanización (CAREY, 2007; 2009; WILKINSON, 2005; STEVENSON, 2007; GONZÁLEZ GONZÁLEZ, 2012). La aparición de nuevas actividades (por ejemplo, la programación y todas las tareas asociadas) o el nuevo peso que adquieren dentro de la economía tareas como la docencia (lo que genera presiones para un incremento de su productividad) conducen a la reproducción de esas viejas batallas en nuevos frentes de combate. Esto opera en un contexto signado por la expansión de la gran industria en extensión y profundidad (en extensión porque alcanza a más ramas; en profundidad porque ramas que ya tenían un proceso de trabajo correspondiente a la gran industria, por medio de la automatización, refuerzan las tendencias propias de ese régimen).

Moraes Neto recupera otro punto relevante a la discusión: a saber, el grado en que las fuerzas productivas capitalistas entran en contradicción con las relaciones sociales de producción y cómo esta contradicción encuentra su máxima expresión a partir del desarrollo de la gran industria. ¿Qué implica la progresiva eliminación del trabajo humano directo en los procesos productivos? Moraes Neto acierta al criticar lo que él denomina un "desvio de olhar" (MORAES NETO, 2003, p. 122) propuesto por Antunes (1988) o Lipietz (1992) al apelar al empleo masivo de trabajo en ciertas actividades como las desarrolladas en el sudeste asiático para esquivar este problema, en la medida que estos procesos no anulan la tendencia central de capital a emplear menos fuerza de trabajo.

Cabe señalar, no obstante, otra contradicción capitalista y una manera en que las relaciones de producción frenan hoy el avance de las fuerzas productivas. El avance de la aplicación de la ciencia a la industria 
es más lento en el capitalismo de lo que sería en el socialismo porque, mientras que de una perspectiva socialista el uso de maquinaria tiene sentido cuando el valor de la misma es menor que el valor del trabajo manual que desplazaría; bajo el capitalismo, su campo de acción es menor porque el capitalista compara el trabajo que implica la producción mecánica, no contra todo el trabajo que absorbe la producción manual, sino solo contra la parte que él paga de la misma, es decir sólo el tiempo de trabajo necesario. A su vez, a medida que el avance de la gran industria genera una masa de sobrepoblación relativa que puede ofrecer su trabajo por debajo de su valor, esto mismo actúa también como desincentivo a la mecanización (MARX, 1999, t. 1, v. 2, pp. 478-9). Al respecto, puede considerarse ilustrativo_el caso de los cartoneros donde incluso se da una reversión técnica al pasarse de la tracción a caballo a la tracción humana del carro de recolección con el correspondiente mayor desgaste físico de los obreros, proceso que es contemporáneo con la ampliación dela sobrepoblación relativa por la expulsión de obreros en otras ramas (VILLANOVA, 2017). Más allá de estas salvedades, que deben ser tenidas en cuenta, la tendencia central de capital a un desplazamiento del trabajo vivo por trabajo muerto de la mano del desarrollo del conocimiento científico se mantiene en pie. Esto no puede despertar nostalgias por formas pasadas de organización del trabajo ${ }^{10}$ que, por otra parte sería inviable recrear. Más bien, debe constituir la base técnica sobre la cual pensar una sociedad liberada de la necesidad del trabajo (donde la drástica reducción de la jornada laboral permita a todas las personas la dedicación a actividades creadoras). Es decir, el desarrollo de la gran industria es la base técnica sobre la cual pensar el socialismo como una sociedad del tiempo libre y de liberación de las potencialidades humanas.

\section{Referencias bibliográficas}

AGLIETTA, M. Regulación y crisis del capitalismo. México: Siglo XXI, 1998.

ANTUNES, R. A rebeldia do trabalho. O confronto operariono ABC paulista: as greves de 1978/80. São Paulo: Ed. Unicamp, 1988.

BIL, D. Descalificados. Proceso de trabajo y clase obrera en la rama gráfica (1880-1940). Buenos Aires: Ediciones RyR, 2007.

. La industria argentina de maquinaria agrícola (1870-1975): evolución y problemas de su desarrollo. Buenos Aires: Instituto de Investigaciones Gino Germani, 2009. Disponble en:

10 Benedito Moraes Neto acertadamente llama a evitar sentir "saudade" del fordismo (MORAES NETO, 2003, pp. 116-7). 
<http://biblioteca.clacso.edu.ar/gsdl/collect/ar/ar030/index/assoc/D4148.dir/ji16.pdf>. Access on: 29/o1/2014. . Proceso de trabajo y luchas obreras en los años' 30: Los trabajadores gráficos. Izquierdas, n. 30, pp. 65-88, 2016.

. Transformaciones en la industria y luchas de los obreros gráficos en Buenos Aires (1878-1940). In: En torno a la imprenta en Buenos Aires. Gobierno de la Ciudad de Buenos Aires, 2018, pp. 247-277.

BRAVERMAN, H. The degradation of work in the Twentieth century. Monthly Review, Madrid, Editorial Revolución, 1983 [1982].

. Trabajo y capital monopolista. México: Nuestro tiempo, 1988.

BURAWOY, M. El consentimiento en la producción. Los cambios del proceso productivo en el capitalismo monopolista. Madrid: Ministerio de Trabajo y Seguridad Social, 1989.

CAREY, M. White-collar proletariat? Braverman, the deskilling/upskilling of social work and the paradoxical life of the agency care manager. Journal of Social Work, v. 7, n. 1, pp. 93-114, 2007.

. It's a Bit Like being a robot or working in a factory': Does Braverman help explain the experiences of State social workers in Britain Since 1971? Organization, v. 16, n. 4, pp. 505-27, 2009.

CUNHA E. P. Braverman, subjetividade e função de direção na produção do valor. Cadernos Ebape.BR, 2014, v. 12, n. 4, pp. 741-55.

ENGELS F. El papel del trabajo en la transformación del mono en hombre. Cali: Andreus, 1975.

GONZÁLEZ GONZÁLEZ N. Estrés en el ámbito laboral de las instituciones de salud: Un acercamiento a narrativas cotidianas. Argumentos (México, DF). 2012 Dec;25(70):171-94.

GORZ, A. Miserias del presente, riqueza de lo posible. Buenos Aires: Paidós, 1998.

GOULD, S. J. La postura hizo al hombre. Razón y Revolución, 1996, n. 2. HARARI, Ianina, Luchas obreras por el proceso de trabajo: el caso de los obreros automotrices argentinos (1959-1976). Trabajo y sociedad, n. 20, pp. 175-192, 2013. Disponible en: <http://www.scielo.org.ar/scielo.php?pid=S1514>$68712013000100012 \&$ script $=$ sci_arttext $>$. Access on: 29/01/2014.

A media máquina. Procesos de trabajo, lucha de clases y competitividad en la industria automotriz argentina (1952-1976). Buenos Aires: Ediciones RyR, 2015.

. Un aporte al debate sobre el fordismo y la cadena de montaje: procesos de trabajo y lucha obrera en el caso automotriz desde una óptica marxista. Verinotio - Revista on-line de Filosofia e Ciências Humanas, Rio das Ostras, v. 25, n. 1, abr. 2019. 
KABAT, M. Del taller a la fábrica. Proceso de trabajo, industria y clase obrera en la rama del calzado (Buenos Aires 1870-1940). Buenos Aires: Ediciones RyR, 2005.

. La industria del calzado: cambios en la organización del trabajo entre 1880 y 1940. Desarrollo económico, v. 47, n. 188, pp. 639-659, 2008.

. El impacto de la comercialización de maquinaria estadounidense sobre la industria argentina del calzado (1903-1920). el@tina Revista electrónica de estudios latinoamericanos, n. 52, 2015. Disponible en: <https://www.redalyc.org/pdf/4964/496450649001.pdf>. Access on: 29/01/2016.

KABAT M; HARARI I. Las comisiones internas bajo el peronismo clásico (Argentina 1946-1955): Conflictos en torno a su accionar y reglamentación. Cuadernos de historia, Santiago, 2014, n. 41, pp. 107-131. Disponible en: $<$ https://scielo.conicyt.cl/scielo.php?pid=So719-

12432014000200005\&script $=$ sci_arttext\&tlng $=\mathrm{en} \geq$. Access on: 29/01/2016.

KABAT, M; LARRALDE, V; FERRER, L.; ESCUDÉ, I. Gran Industria y sobrepoblación relativa. Transformaciones del trabajo y desarrollo de las capas obreras desocupadas en la Argentina durante las tres últimas décadas. II jornadas de Historia Reciente, Necochea, 2004.

LIPIETZ A. Espejismos y milagros: problemas de la industrialización en el Tercer Mundo. Bogotá: Universidad Nacional de Colombia/Tercer Mundo Editores, 1992.

LITTLER, C. R. The labour process debate: a theoretical review 1974-1988. Labour process theory, pp. 46-94, 1990.

LÓPEZ RODRÍGUEZ, R. Sobre cuerpos, máquinas y feminismo. Proceso de producción, proceso de trabajo y valor de uso en la producción de las mercancías "fuerza de trabajo" y "placer sexual" en la rama de la prostitución. Theomai, en prensa.

MARINELLI, N. Drones en el agro: para qué se usan y cómo se eligen. Clarín rural, 25/9/2018, disponible en: <https://www.clarin.com/rural/dron-eligeprestaciones_o_5SVslRt1o.html>. Access on: 29/09/2018.

MARX, Karl. "Cuaderno XIX. Continuación del cuaderno V (las máquinas, etc.)”, en MARX, Karl. Progreso técnico y desarrollo capitalista, Pasado y Presente, México, 1982, pp. 163-6.

. El capital. Buenos Aires: Siglo XXI, 1999.

. Economic and philosophic manuscripts of 1844. Dover Publications.com, 2012. 
MONTGOMERY, D. El control obrero en Estados Unidos. Historia sobre las luchas del trabajo, la tecnología y las luchas obreras. Madrid: Ministerio de Trabajo y Seguridad, 1985.

MORAES NETO, B. R. de. Marx, Taylor, Ford: as forças produtivas em discussão. São Paulo: Editora Brasiliense, 1989.

Século vinte e trabalho industrial. Taylorismo/fordismo, ohnoísmo e automatização em debate. São Paulo: Xamã editora, 2003.

PELÁEZ, P.; HARARI, Ianina. A media máquina. Procesos de trabajo, lucha de clases y competitividad en la industria automotriz argentina (1952-1976). H-industri@: Revista de historia de la industria, los servicios y las empresas en América Latina, n. 20, pp. 128-31, 2017.

INFORMANTE ANÓNIMO, testimonio, 2018, ingeniero rural, ex gerente de compañía líder del sector cerealero en la Argentina, responsable de producción maicera, actual consultor privado de productores cerealeros. Entrevista realizada por Marina Kabat, octubre de 2018.

ROWLINSON, M.; HASSARD, J. Economics, Politics, and Labor Process Theory. Capital and Class, v. 18, n. 2, pp. 65-97, 1994.

ROSSIN, J. Testimonio, 2008, gerente nacional del Area Rural de Manpower, Argentina. Entrevista realizada por Marina Kabat, abril de 2008.

SARTELLI, E. Para comer una Hamburguesa. El estudio de los procesos de trabajo, el debate Braverman y el 'fast food' en Argentina. Razón y Revolución, n. 7, pp. 40-61, 2001. Available at: $<$ http://www.razonyrevolucion.org/textos/revryr/prodetrab/ryr7Sartelli.p df $>$. Access on: 29/01/2014.

. Patrones en la ruta: el conflicto agrario y los enfrentamientos en el seno de la burguesía, marzo-julio de 2008. Buenos Aires: Ediciones RyR, 2008.

SARTELLI, E.; KABAT, M. Las transformaciones recientes del proceso de trabajo en el agro argentino y los cambios concomitantes en las relaciones laborales. História na Fronteira, v. 2, n. 2, pp. 1-15, 2009.

; __ Where did Braverman go wrong? A Marxist response to the politicist critiques. Cadernos Ebape.BR, v. 12, n. 4, 2014.

; __ Argentine industrialization: A critique of the liberal and dependentist schools. Analytical gains of geopolitical Economy, Emerald Group Publishing Limited, pp. 227-54, 2016.

SCHVARZER, J. Comentario al libro de Marina Kabat: Del taller a la fábrica. Proceso de trabajo, industria y clase obrera en la rama del calzado, Buenos Aires, 1870-1940. XX Jornadas de Historia Económica, Mar del Plata, 20/10/2006, disponible en: <http://www.ceics.org.ar/gruposinvestigacion/procesos-de-trabajo/ $\geq$.

SMITH, V. El legado de Braverman. Sociología del trabajo, n. 26, 1996. 
STEVENSON, H. Restructuring teachers' work and trade union responses in England: Bargaining for change? American Educational Research Journal, v. 44, n. 2, pp. 224-51, 2007.

TARDITTI, R. El proceso de trabajo en los frigoríficos: una moderna manufactura. Primeras jornadas interdisciplinarias de estudios agrarios y agroindustriales, Bs. Aires, 1999.

THOMPSON, P. The capitalist labour process: concepts and connections. Capital and Class, 34, pp. 7-14, 2010.

VILLANOVA N. Del «cirujeo» al «cartoneo». Cambios en los procesos de trabajo, condiciones laborales y estructura de la clase obrera: Ciudad de Buenos Aires, 1989-2012. RELET-Revista latinoamericana de estudios del trabajo, n. 34, pp. 161-70, 2017.

WILKINSON, G. Workforce remodelling and formal knowledge: The erosion of teachers' professional jurisdiction in English schools. School Leadership and Management, v. 25, n. 5, pp. 421-439, 2005.

Como citar:

KABAT, Marina. La importancia de la historia para comprender el trabajo y sus transformaciones. Verinotio - Revista on-line de Filosofia e Ciências Humanas, Rio das Ostras, v. 25, n. 1, pp. 28-57, abr. 2019.

Data de envio: 24 fev. 2019

Data de aceite: 28 mar. 2019 